\title{
Surface Embedding of Non-Bipartite $k$-Extendable Graphs
}

\author{
Hongliang $\mathrm{Lu}^{1, *}$ and David G. L. Wang ${ }^{2, *}$ \\ ${ }^{1}$ School of Mathematics and Statistics, Xi'an Jiaotong University, Xi'an, \\ Shaanxi 710049, China \\ 2 School of Mathematics and Statistics 83 MIIT Key Laboratory of \\ Mathematical Theory and Computation in Information Security, \\ Beijing Institute of Technology, Beijing 102400, China
}

Received 23 November 2021; Accepted (in revised version) 13 December 2021

\begin{abstract}
For every surface, we find the minimum number $k$ such that every non-bipartite graph that is embeddable in that surface is not $k$-extendable. In particular, we construct a family of 3-extendable graphs which we call bowtie graphs. This confirms the existence of an infinite number of 3-extendable non-bipartite graphs that are embeddable in the Klein bottle.
\end{abstract}

AMS subject classifications: $05 \mathrm{C} 10,05 \mathrm{C} 70$

Key words: Non-bipartite graph, matching extension, surface embedding.

\section{Introduction}

A matching $M$ of a graph $G$ is said to be extendable if $G$ has a perfect matching containing $M$. A graph is $k$-extendable if it has a matching consisting of $k$ edges and every matching consisting of $k$ edges is extendable, where

$$
1 \leq k \leq(|V(G)|-2) / 2 .
$$

Much attention to the theory of matching extension has been paid since it was introduced by Plummer [17] in 1980. We recommend Lovász and Plummer's book [11] for

*Corresponding author.

Emails: luhongliang@mail.xjtu.edu.cn (H. Lu), glw@bit.edu.cn (G. Wang) 
an excellent survey of the matching theory, and [21,27] for recent progress. Interests in the matching extensions of graphs embedded on surfaces began with the charming result [19] that no planar graph is 3-extendable. We refer the reader to Gross and Tucker's book [5] for basic notions on topological graph theory; see also [2].

Plummer [18] considered the problem of determining the minimum integer $k$ such that every $\Sigma$-embeddable graph is not $k$-extendable. Based on some partial results of Plummer, Dean [3] found the complete answer to this problem.

Theorem 1.1 (Dean, Plummer). Let $\Sigma$ be a surface of characteristic $\chi$. Let $\mu(\Sigma)$ be the minimum integer $k$ such that every $\Sigma$-embeddable graph is not $k$-extendable. Then we have

$$
\mu(\Sigma)= \begin{cases}3, & \text { if } \chi=2, \\ 2+\lfloor\sqrt{4-2 \chi}\rfloor, & \text { otherwise }\end{cases}
$$

Its proof made a heavy use of the Euler contribution technique, which dates back to Lebesgue [8], developed by Ore [14], and flourished by Ore and Plummer [15].

In a previous paper [12], we extended Theorem 1.1 by finding the minimum integer $k$ such that there is no $\Sigma$-embeddable $(n, k)$-graphs, where an $(n, k)$-graph is a graph whose subgraph obtained by removing any $n$ vertices is $k$-extendable. This paper continues the study of this embeddable-extendable type of problems. We dig a little deeper by concentrating on non-bipartite graphs. Here is our main result.

Theorem 1.2. Let $\Sigma$ be a surface of characteristic $\chi$. Let $\mu^{\prime}(\Sigma)$ to be the minimum integer $k$ such that every $\Sigma$-embeddable non-bipartite graph is not $k$-extendable. Then we have

$$
\mu^{\prime}(\Sigma)= \begin{cases}4, & \text { if } \chi \in\{-1,0\} \\ 3, & \text { if } \chi=2, \\ \lfloor(7+\sqrt{49-24 \chi}) / 4\rfloor, & \text { otherwise. }\end{cases}
$$

Non-bipartite graphs differ from bipartite graphs in many aspects, even if we are concerned with only matching problems. For instance, König theorem states that the maximum size of a matching in a bipartite graph equals the minimum size of a vertex cover; see Rizzi [24] for a short proof. Taking a triangle as the graph under consideration, one may see immediately that non-bipartite graphs do not admit this beautiful property in general.

Another example is on the algorithmic complexity. Lakhal and Litzler [7] discovered a polynomial-time algorithm for the problem of finding the extendability of a bipartite graph. It is still unknown that whether the same extendability problem for non-bipartite graphs can be solved in polynomial time or not; see Plummer [20]. 
The sharp distinction between the appearances of Eqs. (1.1) and (1.2), also supports the above difference between bipartite and non-bipartite graphs in the theory of matching extensions.

A big part of the proof of Theorem 1.2 is to show the 3-extendability of some so-called bow-tie graphs. We think the family of bow-tie graphs is interesting also on its own right. We will confirm the infinity of the number of 3-extendable graphs which can be embedded onto the Klein bottle, and which are non-bipartite. An infinity number of such, but bipartite, graphs, were constructed recursively by Aldred, Kawarabayashi, and Plummer [1].

This paper is organized as follows. In the next section we list necessary notion and notation, as well as necessary known results in the field of surface embedding and matching extension of graphs. The stand-alone Section 3 is devoted to the extendability of the two families of Cartesian product graphs of paths and cycles, and of the bow-tie graphs denoted as $C_{6} \bowtie P_{n}$ for odd $n \geq 5$. We also pose a conjecture for the 3-extendability of the general bow-tie graphs. In Section 4 we establish Theorem 1.2 with the aid of these extendability results.

\section{Preliminaries}

This section contains an overview of necessary notion and notation. Let $G=(V, E)$ be a simple graph. We denote the number $|V(G)|$ of vertices by $|G|$ for short. Denote by $\delta(G)$ the minimum degree of $G$, and by $\kappa(G)$ the connectivity.

\subsection{The surface embedding}

A surface is a connected compact Hausdorff space which is locally homeomorphic to an open disc in the plane. If a surface $\Sigma$ is obtained from the sphere by adding some number $g$ of handles (resp. some number $\tilde{g}$ of cross-caps), then $\Sigma$ is said to be orientable of genus $g$ (resp. non-orientable of non-orientable genus $\tilde{g}$ ). We shall follow the usual convention of denoting the surface of genus $h$ (resp. non-orientable genus $k$ ) by $S_{h}$ (resp. $N_{k}$ ).

A 2-cell embedding of a graph $G$ onto a surface is a drawing of the graph $G$ on the surface such that the edges of $G$ cross only at the vertices of $G$, and that every face is homeomorphic to an open disk. In this paper, we wording "embedding" always means 2-cell embedding. We say that a graph $G$ is $\Sigma$-embeddable if there exists an embedding of the graph $G$ on the surface $\Sigma$. The minimum value $g$ such that $G$ is $S_{g}$-embeddable is said to be the genus of $G$, denoted $g(G)$. Any embedding of $G$ on $S_{g(G)}$ is said to be a minimal (orientable) embedding. Similarly, the minimum value $\tilde{g}$ such that $G$ is $N_{\tilde{g}}$-embeddable is said to be the non-orientable genus of $G$, 
denoted $\tilde{g}(G)$. For a general surface $\Sigma$, let $g(\Sigma)$ be the genus of $\Sigma$. The Euler characteristic $\chi(\Sigma)$ is defined by

$$
\chi(\Sigma)= \begin{cases}2-2 g(\Sigma), & \text { if } \Sigma \text { is orientable, } \\ 2-\tilde{g}(\Sigma), & \text { if } \Sigma \text { is non-orientable. }\end{cases}
$$

Any embedding of $G$ on $N_{\tilde{g}(G)}$ is said to be a minimal (non-orientable) embedding. Working on minimal embeddings, one should notice the following two fundamental results, which are due to Youngs [26] and Parsons et al. [16] respectively.

Theorem 2.1 (Youngs). Every minimal orientable embedding of a graph is 2-cell.

Theorem 2.2 (Parson, Pica, Pisanski and Ventre). Every graph has a minimal non-orientable embedding which is 2-cell.

The formula of non-orientable genus of complete graphs was found by Franklin [4] in 1934 for $K_{7}$, and by Ringel [22] in 1954 for the other $K_{n}$. Early contributors include Heawood, Tietze, Kagno, Bose, Coxeter, Dirac, and so on; see [22]. The more difficult problem of finding the genus of complete graphs has been explored by Heffter, Ringel, Youngs, Gustin, Terry, Welch, Guy, Mayer, and so on. A short history can be found in the famous work [23] of Ringel and Youngs in 1968, who settled the last case. These formulas are as follows.

Theorem 2.3. Let $n \geq 5$. We have

1. $\tilde{g}\left(K_{7}\right)=3$ and $\tilde{g}\left(K_{n}\right)=\lceil(n-3)(n-4) / 6\rceil$ when $n \neq 7$,

2. $g\left(K_{n}\right)=\lceil(n-3)(n-4) / 12\rceil$.

\subsection{The Euler contribution}

Let $G \rightarrow \Sigma$ be an embedding of a graph $G$ on the surface $\Sigma$. Euler's formula states that

$$
|G|-e+f=\chi(G),
$$

where $e$ is the number of edges of $G$, and $f$ is the number of faces in the embedding. Let $x_{i}$ denote the size of the $i$ th face containing $v$, i.e., the length of its boundary walk. The Euler contribution of $v$ is defined to be

$$
\Phi(v)=1-\frac{d(v)}{2}+\sum_{i} \frac{1}{x_{i}}
$$


where the sum ranges over all faces containing $v$. One should keep in mind that a face may contribute more than one angle to a vertex. This can be seen from the embedding of $K_{5}$ on the torus. From Euler's formula, in any embedding of a connected graph $G$, we have

$$
\sum_{v} \Phi(v)=\chi(\Sigma)
$$

Thus there exists a vertex $v$ such that

$$
\Phi(v) \geq \frac{\chi(\Sigma)}{|G|} .
$$

Such a vertex is said to be a control point of the embedding. This definition implies the following lemma immediately, see also [18, Lemma 2.5] or [3, Lemma 2.5] for its proof.

Lemma 2.1. Let $G$ be a connected graph of at least 3 vertices. Let $G \rightarrow \Sigma$ be an embedding. Let $v$ be a control point which is contained in $x$ triangular faces. Then we have

$$
\frac{d(v)}{6} \leq \frac{d(v)}{4}-\frac{x}{12} \leq 1-\frac{\chi(\Sigma)}{|G|}
$$

\subsection{The matching extension}

Let $G$ be a graph and $k \geq 0$. A $k$-matching of $G$ is a collection of $k$ pairwise disjoint edges. Perfect matchings are $|G| / 2$-matchings. A near perfect matching of the graph $G$ is a perfect matching of the graph $G-v$ for some vertex $v$ of $G$. The most basic result for perfect matchings is Tutte's theorem [25].

Theorem 2.4 (Tutte). A graph $G$ has a perfect matching if and only if for every vertex subset $S$, the subgraph $G-S$ has at most $|S|$ connected components with an odd number of vertices.

The graph $G$ is said to be $k$-extendable if

- it has a perfect matching, and

- for any $k$-matching $M$, the graph $G$ has a perfect matching containing $M$.

The following basic property on the connectivity of extendable graphs can be found in [17].

Theorem 2.5 (Plummer). Let $k \geq 0$ and let $G$ be a connected $k$-extendable graph. Then $G$ is $(k+1)$-connected and $\delta(G) \geq k+1$. 
Liu and $\mathrm{Yu}[9]$ found the following result for the extendability of Cartesian product graphs.

Theorem 2.6 (Liu and $\mathrm{Yu}$ ). Let $G_{1}$ be a k-extendable graph and $G_{2}$ be a connected graph. Then the Cartesian product $G_{1} \times G_{2}$ is $(k+1)$-extendable.

Györi and Plummer [6] gave the following nice generalization.

Theorem 2.7 (Györi and Plummer). The Cartesian product of a $k$-extendable graph and an l-extendable graph is $(k+l+1)$-extendable.

Plummer [19] also gave the famous result that no planar graph is 3-extendable. The next deeper result is due to Lou and $\mathrm{Yu}$ [10, Theorem 7]; see also [27, Chapter 6].

Theorem 2.8 (Lou and $\mathrm{Yu}$ ). If $G$ is a $k$-extendable graph of order at most $4 k$, then either $G$ is bipartite or the connectivity $\kappa(G)$ of $G$ is at least $2 k$.

We also need the following result, whose proof can be found in $[3,12]$.

Lemma 2.2. Let $k \geq 1$. Let $G$ be a connected $k$-extendable graph embedded on a surface $\Sigma$. Let $v$ be a vertex of $G$ which is contained in $x$ triangular faces in the embedding. Then we have

$$
d(v) \geq \begin{cases}k+1+\lceil x / 2\rceil, & \text { if } x \leq 2 k-2 \\ 2 k+1, & \text { if } x \geq 2 k-1\end{cases}
$$

\section{The matching extension of special product graphs}

In this section, we shall establish the 3-extendability for some special graphs, which will be used in handling some sporadic cases in proving Theorem 1.2.

Denote by $P_{m}$ the path having $m$ vertices. Denote by $P_{m} \times P_{n}$, as usual, the Cartesian product graph of the paths $P_{m}$ and $P_{n}$. We label its vertices by $v_{i, j}$ (or by $v_{i j}$ if there is no confusion), from the northwest to the southeast, where $i \in[m]$ and $j \in[n]$. We use the notation $R_{i}$ to denote the vertex set $\left\{v_{i 1}, \cdots, v_{i n}\right\}$ of the $i$-th row; and use the notation $T_{j}$ to denote the vertex set $\left\{v_{1 j}, \cdots, v_{m j}\right\}$ of the $j$-th column. We say that any edge in a row is horizontal, and that any edge in a column is vertical. For convenience, we consider the first subscript $i$ of the notation $v_{i j}$ as modulo $m$, and consider the second subscript $j$ as modulo $n$, i.e.,

$$
v_{i+k m, j+h n}=v_{i j} \quad \text { for all } k, h \in \mathbb{Z} \text {. }
$$




\begin{tabular}{|c|c|c|c|c|}
\hline$v_{11}$ & $v_{12}$ & $v_{13}$ & $v_{14}$ & $v_{15}$ \\
\hline$v_{21}$ & $v_{22}$ & $v_{23}$ & $v_{24}$ & $v_{25}$ \\
\hline$v_{31}$ & $v_{32}$ & $v_{33}$ & $v_{34}$ & $v_{35}$ \\
\hline$v_{41}$ & $v_{42}$ & $v_{43}$ & $v_{44}$ & $v_{45}$ \\
\hline$v_{51}$ & $v_{52}$ & $v_{53}$ & $v_{54}$ & $v_{55}$ \\
\hline$v_{61}$ & $v_{62}$ & $v_{63}$ & $v_{64}$ & $v_{65}$ \\
\hline$v_{11}$ & $v_{12}$ & $v_{13}$ & $v_{14}$ & $v_{15}$ \\
\hline
\end{tabular}

Figure 1: The bow-tie graph $C_{6} \bowtie P_{5}$ is $N_{2}$-embeddable.

It follows that $R_{i+m}=R_{i}$ for all $i$, and that $T_{j+n}=T_{j}$ for all $j$. Denote by $C_{n}$ the cycle having $n$ vertices. We use the same way to label the vertices of the graphs $P_{m} \times C_{n}$ and $C_{m} \times C_{n}$.

For any positive integers $m$ and $n$, we define the bow-tie graph $C_{m} \bowtie P_{n}$ to be the graph obtained from the graph $C_{m} \times P_{n}$ by adding the edges $v_{i 1} v_{m+2-i, n}$ for all $i \in[m]$. From Fig. 1, it is easy to see that the graph $C_{m} \bowtie P_{n}$ is $N_{2}$-embeddable.

In the subsequent three subsections, we will explore the matching extension of the following graphs respectively:

$$
P_{m} \times C_{n}, \quad C_{m} \times C_{n}, \quad \text { and } \quad C_{m} \bowtie P_{n} .
$$

Precisely speaking, we will show that the graph $P_{m} \times C_{n}$ is 2-extendable, and the other two graphs are 3 -extendable, subject to some natural conditions on the integers $m$ and $n$.

Here we describe a combinatorial idea, which will be adopted in all the proofs uniformly. Let $G$ be a graph with a matching $M$. We say that $G$ is separable by a subgraph $G^{\prime}$ (with respect to $M$ ), if

- the matching $M$ has at least one edge in the subgraph $G^{\prime}$; and

- no edge of the matching $M$ has ends in both of the subgraphs $G^{\prime}$ and $G-V\left(G^{\prime}\right)$.

We call the subgraph $G^{\prime}$ an $M$-separator of $G$, if

- the subgraph $G^{\prime}$ has a perfect matching containing the edge set $M \cap E\left(G^{\prime}\right)$; and 
- the subgraph $G-V\left(G^{\prime}\right)$ has a perfect matching containing the edge set $M \cap$ $E\left(G-V\left(G^{\prime}\right)\right)$.

In particular, the subgraph $G-V\left(G^{\prime}\right)$ has a perfect matching even if the set $M \cap$ $E\left(G-V\left(G^{\prime}\right)\right)$ is empty. From the above definition, it is direct to see that the extendability of a matching $M$ can be confirmed by finding an $M$-separator. We call this approach the separator method. We will use it uniformly by choosing the separator to be a subgraph induced by consecutive rows or columns.

\subsection{The 2-extendability of the graph $P_{m} \times C_{n}$}

This subsection is devoted to establish the following result. It is basic and will be used in the proof of Lemma 3.1 and Theorems 1.2 and 3.2.

Theorem 3.1. Let $m, n \geq 4$. The Cartesian product graph $P_{m} \times C_{n}$ is 2-extendable if and only if the integer $m$ or $n$ is even.

Proof. The necessity is clear from the definition. When $n$ is even, the cycle $C_{n}$ is 1-extendable. Thus the sufficiency is true from Theorem 2.6. It suffices to show the sufficiency for odd $n$.

Let $n \geq 5$ be an odd integer. Then the integer $m$ is even. Let $G$ be the graph $P_{m} \times C_{n}$, with a 2-matching $M=\left\{e_{1}, e_{2}\right\}$. Note that every column of the graph $G$ is isomorphic to the path $P_{m}$, which has a perfect matching. We will adopt the separator method by finding some columns, whose induced subgraph has a perfect matching containing the matching $M$. We have 3 cases to treat.

Case 1. There are two disjoint pairs of adjacent columns, such that one pair contains the vertex set $V\left(e_{1}\right)$, and the other pair contains the vertex set $V\left(e_{2}\right)$. Since the subgraph induced by any two adjacent columns is 1-extendable, the four columns form an $M$-separator.

Case 2. The vertex set $V(M)$ is contained in two adjacent columns, and Case 1 does not occur. Then the two adjacent columns form an $M$-separator. In fact, when both the edges $e_{1}$ and $e_{2}$ are vertical and in distinct columns, the previous possibility happens, a contradiction. In other words, either the vertex set $V(M)$ is contained in one column, or one of the edges in the matching $M$ is horizontal.

Case 3. Otherwise, the vertex set $V(M)$ intersects with exactly three consecutive columns, and both the edges $e_{1}$ and $e_{2}$ are horizontal.

We proceed by induction on $m$. For $m=4$, we have 2 sub-cases to treat.

Case 3-1 Assume that the edges in the matching $M$ lie in Row 1 and Row 2, or in Row 1 and Row 3. Since every row is isomorphic to a cycle, we can suppose 
without loss of generality that

$$
e_{1}=v_{11} v_{12} \quad \text { and } \quad e_{2} \in\left\{v_{22} v_{23}, v_{32} v_{33}\right\} .
$$

In this case, the subgraph $G\left[T_{1}, T_{2}, T_{3}, T_{4}\right]$ has the perfect matching

$$
\left\{v_{11} v_{12}, v_{13} v_{14}, v_{22} v_{23}, v_{32} v_{33}, v_{41} v_{42}, v_{43} v_{44}, v_{21} v_{31}, v_{24} v_{34}\right\},
$$

which contains the matching $M$, see Fig. 2 .

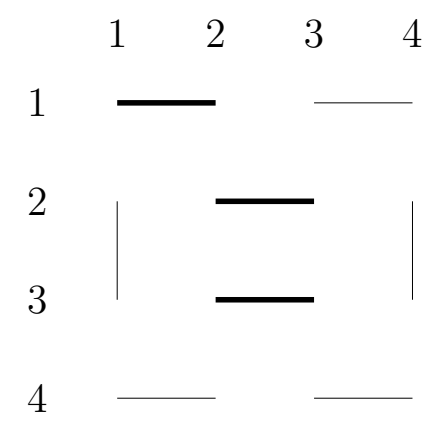

Figure 2: The extension of the matching $M$ for Case 3-1.

Case 3-2 Otherwise, the edges in the matching $M$ lie in Row 1 and Row 4, or in Row 2 and Row 3. We can suppose that

$$
M=\left\{v_{11} v_{12}, v_{42} v_{43}\right\} \quad \text { or } \quad M=\left\{v_{21} v_{22}, v_{32} v_{33}\right\} .
$$

In this case, the subgraph $G\left[T_{1}, T_{2}, T_{3}\right]$ has the perfect matching

$$
\left\{v_{11} v_{12}, v_{21} v_{22}, v_{13} v_{23}, v_{31} v_{41}, v_{32} v_{33}, v_{42} v_{43}\right\}
$$

which contains the matching $M$, see Fig. 3 .

This completes the proof for $m=4$.

Now we can suppose that $m \geq 6$, and that the graph $P_{m-2} \times C_{n}$ is 2-extendable. Note that the subgraph induced by any two adjacent rows has a perfect matching. By induction, we are done if the matching $M$ shares no vertices with the first two rows. For the same reason, we are done if $M$ shares no vertices with the last two rows. Since $m \geq 6$, we can suppose that the horizontal edge $e_{1}$ is in the first two rows, and that the horizontal edge $e_{2}$ is in the last two rows. On one hand, the subgraph $G\left[R_{1}, R_{2}\right]$ is isomorphic to the graph $P_{2} \times C_{n}$, which is 1-extendable. On the other hand, the subgraph $G-R_{1}-R_{2}$ is isomorphic to $P_{m-2} \times C_{n}$, which is 2-extendable by induction hypothesis. Hence, the subgraph $G\left[R_{1}, R_{2}\right]$ is an $M$ separator. This completes the proof. 


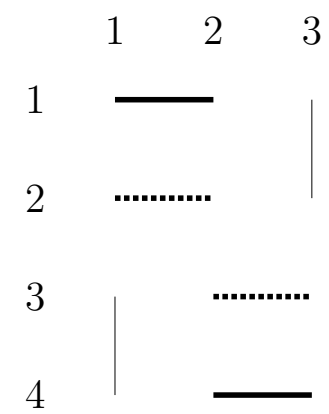

Figure 3: The extension of the matching $M$ for Case 3-2.

\subsection{The 3-extendability of the graph $C_{m} \times C_{n}$}

In this subsection we study the extendability of the graph $C_{m} \times C_{n}$, which will be used in the proof of Theorem 1.2.

A necessary condition for the graph $C_{m} \times C_{n}$ to have a perfect matching is that one of the integers $m$ and $n$ is even. By symmetry, we can suppose that the integer $m$ is even. In virtue of Theorem 2.7, the graph $C_{m} \times C_{n}$ is 3 -extendable if the integer $n$ is also even. Therefore, we can suppose that $n$ is odd. The following lemma will be used for several times.

Lemma 3.1. Let $m \geq 6$ be an even integer, and let $n \geq 5$ be an odd integer. Let $G$ be the graph $C_{m} \times C_{n}$, with a 3-matching $M$. Then the matching $M$ is extendable if $G$ is separable by

(i) a subgraph $G\left[R_{i}, R_{i+1}\right]$ for some $i \in[m]$, which contains exactly one edge of the matching $M$; or

(ii) a subgraph $G\left[T_{j}, T_{j+1}\right]$ for some $j \in[n]$, which contains one or two edges of the matching $M$.

Proof. We prove the validities of Condition (i) and Condition (ii) separately. Let $i \in[m]$ and $j \in[n]$.

(i) The graph $G\left[R_{i}, R_{i+1}\right]$ is isomorphic to $P_{2} \times C_{n}$, which is 1-extendable. The subgraph $G-R_{i}-R_{i+1}$ is isomorphic to $P_{m-2} \times C_{n}$. Since $m-2 \geq 4$, the graph $G-$ $R_{i}-R_{i+1}$ is 2-extendable by Theorem 3.1. Hence, the matching $M$ is extendable in $G$.

(ii) The graph $G\left[T_{j}, T_{j+1}\right]$ is isomorphic to $C_{m} \times P_{2}$, and $G-T_{j}-T_{j+1}$ is isomorphic to $C_{m} \times P_{n-2}$. Both of them are 2-extendable by Theorem 2.7. Hence, the matching $M$ is extendable in the graph $G$. 
Here is the main result of this subsection.

Theorem 3.2. Let $m \geq 6$ be an even integer, and let $n \geq 5$ be an odd integer. Then the Cartesian product graph $C_{m} \times C_{n}$ is 3-extendable.

Proof. Let $m \geq 6$ be an even integer, and let $n \geq 5$ be an odd integer. Let $G=C_{m} \times C_{n}$, with a 3 -matching $M=\left\{e_{1}, e_{2}, e_{3}\right\}$. In order to show that $M$ is extendable, it suffices to find

- a row index $i^{*}$ such that the subgraph $G\left[R_{i^{*}}, R_{i^{*}+1}\right]$ is an $M$-separator, or

- a column index $j^{*}$ such that the subgraph $G\left[T_{j^{*}}, T_{j^{*}+1}\right]$ is an $M$-separator.

Let $h$ be the number of horizontal edges in the matching $M$. Then we have $h \in$ $\{0,1,2,3\}$. We treat these 4 cases individually.

Case 1. $h=0$, that is, all edges in $M$ are vertical. Note that each column of the graph $G$ is isomorphic to the cycle $C_{m}$, which is 1-extendable. If the 3 edges in $M$ are in distinct columns, we are done immediately. If they are in the same column, then that column together with one of its adjacent columns form an $M$-separator. Otherwise, we can suppose that Column $j$ contains the edges $e_{1}$ and $e_{2}$, but not the edge $e_{3}$. By virtue of Lemma 3.1, we can take $j^{*}=j$ if $e_{3}$ is not in Column $(j+1)$; and take $j^{*}=j+1$ otherwise.

Case 2. $h=1$. In this case, we can suppose that $e_{1}$ is horizontal, and that $e_{2}$ and $e_{3}$ are vertical. Since each row is isomorphic to a cycle, we can further suppose that $e_{1}=v_{i 1} v_{i 2}$ and it intersects with the first two columns.

If at most one of the edges $e_{2}$ and $e_{3}$ is in the first two columns, then we can take $j^{*}=1$ by Lemma 3.1. Otherwise, both of them are in the first two columns. If the vertex set $V(M)$ misses Row $(i+1)$, then we can take $i^{*}=i$ by Lemma 3.1. For the same reason, we can take $i^{*}=i-1$ if $V(M)$ misses Row $(i-1)$. Otherwise, one of the edges $e_{2}$ and $e_{3}$ is immediately above the horizontal edge $e_{1}$, and the other is immediately below $e_{1}$. In this case, we can take $i^{*}=i+1$ by Lemma 3.1.

Case 3. $h=2$. We can suppose that $e_{1}$ and $e_{2}$ are horizontal, and that $e_{3}$ is vertical. Furthermore, we can suppose that the horizontal edge $e_{1}$ intersects with the first two columns, the horizontal edge $e_{2}$ intersects with Column $j$ and Column $(j+1)$, and that the vertical edge $e_{3}=v_{p q} v_{p+1, q}$, where $p \in[m]$ and $q \in[n]$.

If $j=1$, to wit, the horizontal edge $e_{2}$ lies below the edge $e_{1}$. In this case, we can take $j^{*}=1$. In fact, since $e_{3}$ is vertical, the graph $G$ is separable by the subgraph $G\left[T_{1}, T_{2}\right]$. On the other hand, it is easy to see that both of the subgraphs

$$
G\left[T_{1}, T_{2}\right]-V\left(e_{1} \cup e_{2}\right) \quad \text { and } \quad G-T_{1}-T_{2}
$$


are 1-extendable.

If $j \geq 3$, then the graph $G$ is separable by the first two columns with respect to the matching $M$. In this case, we can also take $j^{*}=1$, by using Lemma 3.1.

Otherwise, we have $j=2$, that is, the vertex set of the horizontal edges $e_{1}$ and $e_{2}$ intersects with exactly the first three columns.

- If $q \in$ [3], i.e., the edge $e_{3}$ is also contained in the subgraph $G\left[T_{1}, T_{2}, T_{3}\right]$, then we can take $i^{*}=p$. In fact, the subgraph $G^{\prime}=G\left[R_{p}, R_{p+1}\right]$ contains the vertical edge $e_{3}$, and possibly one of the edges $e_{1}$ and $e_{2}$. In any case, the matching $M \cap E\left(G^{\prime}\right)$ is extendable in the subgraph $G^{\prime}$.

- If $q \geq 4$, i.e., the vertical edge $e_{3}$ has empty intersection with the first three columns. In this case, the subgraph $G\left[T_{q}\right]$ is an $M$-separator. In fact, the subgraph $G\left[T_{q}\right]$, which contains the edge $e_{3}$, is isomorphic to the cycle $C_{m}$, which is 1-extendable. On the other hand, the subgraph $G-T_{q}$ is isomorphic to the graph $C_{m} \times P_{n-1}$, i.e., the graph $P_{n-1} \times C_{m}$. Since $n-1 \geq 4$, it is 2 extendable by Theorem 3.1.

Case 4. $h=3$. If all edges in $M$ lie in the same row, by Lemma 3.1, we can take $j^{*}=j$ for any edge $v_{i j} v_{i, j+1} \in M$. Otherwise, there exists a row $R_{i}$ containing exactly one edge in $M$ such that one of its adjacent rows has no edges in $M$. In other words, either

$$
R_{i-1} \cap V(M)=\emptyset \quad \text { or } \quad R_{i+1} \cap V(M)=\emptyset .
$$

By Lemma 3.1, we can take $i^{*}=i-1$ in the former case, and $i^{*}=i$ in the latter case. This completes the proof.

We remark that the graph $C_{4} \times C_{n}$ is not 3 -extendable when $n$ is odd. This can be seen from the fact that the particular 3-matching

$$
M=\left\{v_{11} v_{12}, v_{22} v_{32}, v_{31} v_{41}\right\}
$$

is not extendable, see Fig. 4. Define

$$
U=\left\{v_{i, 2 j}: \quad i \in\{1,3\}, 2 \leq j \leq(n-1) / 2\right\} \cup\left\{v_{i, 2 j+1}: i \in\{2,4\}, 1 \leq j \leq(n-1) / 2\right\} .
$$

We have $|U|=2 n-4$. Note that the subgraph $G-V(M)-U$ consists of $2 n-2$ isolated vertices. By Theorem 2.4, the subgraph $G-V(M)$ has no perfect matchings. 


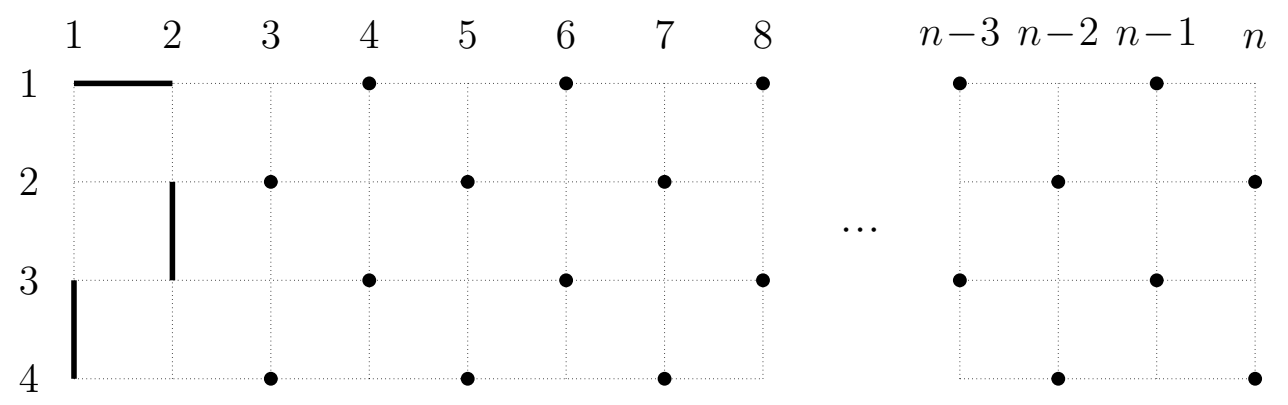

Figure 4: The graph $C_{4} \times C_{n}$ is not 3 -extendable when $n$ is odd.

\subsection{The 3-extendability of the graph $C_{6} \bowtie P_{n}$ for odd $n \geq 5$}

In this subsection, we will show the 3-extendability of the graph $C_{6} \bowtie P_{n}$ for odd integers $n \geq 5$. It is easy to see that $C_{6} \bowtie P_{n}$ can be drawn as in Fig. 5, which is symmetric up and down. For convenience, we rename the vertices in the following way:

$$
\begin{array}{lll}
v_{1 i}=h_{i}, & v_{6 i}=q_{i}, & v_{2 i}=q_{i+n}, \\
v_{4 i}=h_{i}^{\prime}, & v_{5 i}=q_{i}^{\prime}, & v_{3 i}=q_{i+n}^{\prime},
\end{array}
$$

and use capital letters to denote vertex subsets as follows:

$$
\begin{array}{lll}
H=\left\{h_{i}: i \in[n]\right\}, & Q=\left\{q_{j}: j \in[2 n]\right\}, & J=H \cup Q, \\
H^{\prime}=\left\{h_{i}^{\prime}: i \in[n]\right\}, & Q^{\prime}=\left\{q_{j}^{\prime}: j \in[2 n]\right\}, & J^{\prime}=H^{\prime} \cup Q^{\prime} .
\end{array}
$$

Let us keep in mind that the subscript $i$ in the symbols $h_{i}$ are considered modulo $n$, and the subscript $i$ in the symbols $q_{i}$ are modulo $2 n$, namely,

$$
h_{i+n}=h_{i} \quad \text { and } \quad q_{j+2 n}=q_{j} \quad \text { for all integers } i \text { and } j .
$$

Theorem 3.3. Let $n \geq 5$ be an odd integer. Then the bow-tie graph $C_{6} \bowtie P_{n}$ is 3-extendable.

Proof. Let $G=C_{6} \bowtie P_{n}$, where $n$ is an odd integer and $n \geq 5$.

Let $M_{0}$ be a 3-matching of the graph $G$. We call an edge of $M_{0}$ faithful if it is an edge of the subgraph $G[J]$; co-faithful if it is an edge of the subgraph $G\left[J^{\prime}\right]$; and unfaithful otherwise, i.e., if it is an edge of the form $q_{j} q_{j}^{\prime}$ for some $j \in[2 n]$. Correspondingly, we call a vertex of the matching $M_{0}$ faithful (resp. co-faithful, unfaithful) if it is a vertex of a faithful (resp. co-faithful, unfaithful) edge. 


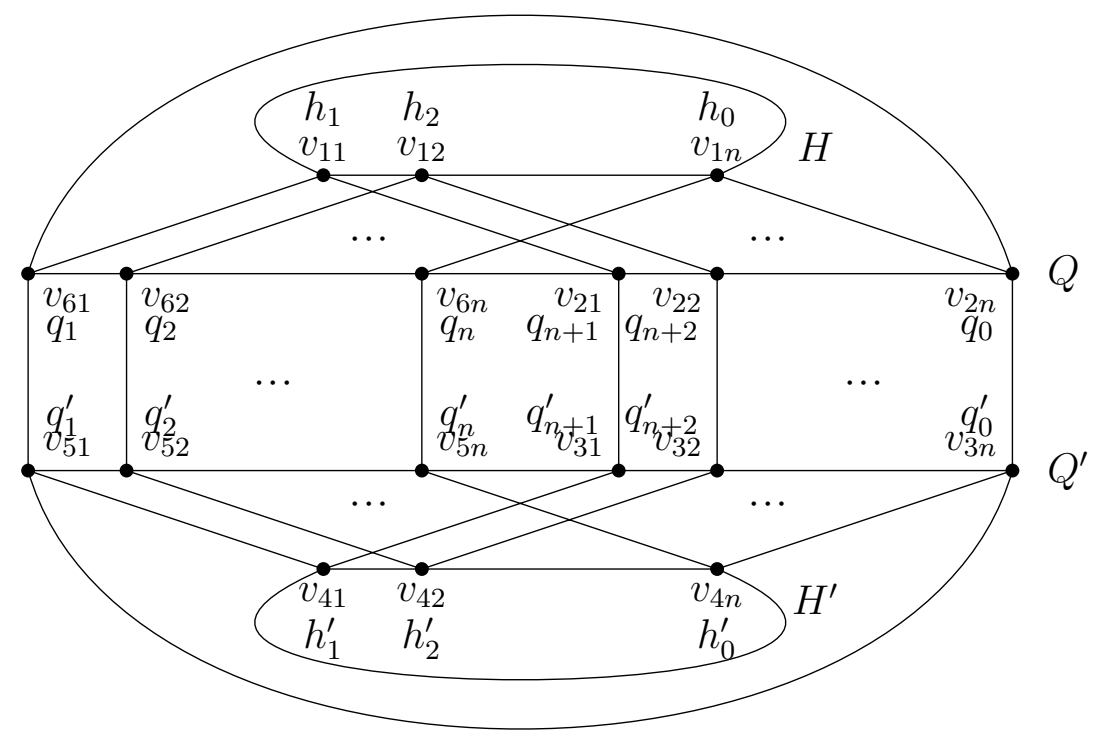

Figure 5: The graph $C_{6} \bowtie P_{n}$.

Suppose that the matching $M_{0}$ has $x$ faithful edges, $y$ unfaithful edges, and $z$ co-faithful edges. Then we have $x+y+z=3$. By the symmetry of the graph $G$, we can suppose that $x \geq z$. Then we have $z=0$ or $z=1$. For each of them, we will construct a perfect matching of the graph $G$ which extends the matching $M_{0}$.

The following lemma serves for Lemma 3.3, by which we can solve the case $z=0$. The other case $z=1$ can be divided into the cases

$$
(x, y, z)=(1,1,1) \quad \text { and } \quad(x, y, z)=(2,0,1) .
$$

We will handle them by Lemmas 3.4 and 3.5 respectively.

Lemma 3.2. Every 3-matching of the subgraph $G[J]$ can be extended to a matching covering the vertex set $H$.

Proof. Suppose that the claim is false. Let $M_{0}$ be a 3-matching which is not extendable in this way. Let $\widetilde{M}$ be an extension of $M_{0}$, which covers the maximum number of vertices in the set $H$. Without loss of generality, we can suppose that $h_{1} \notin V(\widetilde{M})$. Then $q_{1}, q_{n+1} \in V\left(M_{0}\right)$, and we can write

$$
M_{0}=\left\{q_{0} q_{1}, e_{2}, e_{3}\right\}
$$

where $e_{2} \in\left\{q_{n} q_{n+1}, q_{n+1} q_{n+2}\right\}$. We will find a contradiction by constructing an extension of $M_{0}$, which covers $H$. It suffices to find a matching $M$ of the subgraph 
$G[H]-V\left(M_{0}\right)$ such that the subgraph $G[H]-V\left(e_{3}\right)-V(M)$ consists of paths of even orders. We proceed according to the number of vertices in $H$ covered by the edge $e_{3}$.

Case 1. If $V\left(e_{3}\right) \cap H=\emptyset$, then we can define

$$
M= \begin{cases}\left\{h_{2} q_{2}\right\}, & \text { if } q_{2} \notin V\left(e_{3}\right), \\ \left\{h_{n-1} q_{n-1}\right\}, & \text { otherwise. }\end{cases}
$$

Case 2. If $\left|V\left(e_{3}\right) \cap H\right|=1$, then we can define $M=\emptyset$.

Case 3. If $\left|V\left(e_{3}\right) \cap H\right|=2$, then we have $e_{3}=h_{i} h_{i+1}$, where $i \in\{2, \cdots, n-1\}$. We can define

$$
M= \begin{cases}\left\{h_{n-1} q_{n-1}\right\}, & \text { if } i \text { is even and } i \neq n-1, \\ \left\{h_{2} q_{2}\right\}, & \text { if } i \text { is odd, } \\ \left\{h_{3} q_{3}\right\}, & \text { if } i=n-1 .\end{cases}
$$

This proves Lemma 3.2.

Here is the lemma by using which the case $z=0$ can be solved.

Lemma 3.3. Suppose that the matching $M_{0}$ has no co-faithful edges. Then the subgraph $G[J]$ has a matching $M$ such that $M$ covers both the faithful edges and the vertex set $H$, and that $M$ misses every unfaithful vertex.

Proof. We proceed according to the number $x$ of faithful edges. The case $x=3$ is Lemma 3.2.

When $x=2$, let $q_{j} q_{j}^{\prime}$ be the unfaithful edge, where $j \in[2 n]$. Assume that the vertex $q_{j-1}$ is uncovered by the matching $M_{0}$. By Lemma 3.2, the 3-matching $\left(M_{0}-q_{j} q_{j}^{\prime}\right) \cup\left\{q_{j-1} q_{j}\right\}$ can be extended to a matching $M_{1}$, which covers the set $H$. Then the matching $M_{1}-q_{j-1} q_{j}$ is a desired one. For the same reason, Lemma 3.3 holds true if the vertex $q_{j+1}$ is uncovered by $M_{0}$. Now, we can suppose that both the vertices $q_{j-1}$ and $q_{j+1}$ are covered by $M_{0}$. By Lemma 3.2, the matching

$$
M_{2}=\left(M_{0}-q_{j} q_{j}^{\prime}\right) \cup\left\{h_{j} q_{j+n}\right\}
$$

can be extended to a matching, say, $M_{2}^{\prime}$, which covers the set $H$. Since all the three neighbors $q_{j-1}, q_{j+1}$ and $h_{j}$, of the vertex $q_{j}$ in the subgraph $G[J]$, are in the matching $M_{2}$ which misses the vertex $q_{j}$, we infer that the unfaithful vertex $q_{j}$ is not covered by $M_{2}^{\prime}$. Therefore, $M_{2}^{\prime}$ is a desired one.

When $x=1$, we can represent the matching $M_{0}$ as

$$
M_{0}=\left\{e_{1}, q_{j} q_{j}^{\prime}, q_{k} q_{k}^{\prime}\right\}
$$


where $1 \leq j<k \leq 2 n$. If the vertices $q_{j}$ and $q_{k}$ are not adjacent in the subgraph $G[Q]$, then there exist two distinct vertices $u$ and $w$ such that

$$
u \in\left\{q_{j-1}, q_{j+1}\right\} \backslash V\left(e_{1}\right) \text { and } w \in\left\{q_{k-1}, q_{k+1}\right\} \backslash V\left(e_{1}\right) .
$$

By Lemma 3.2, the matching $\left\{u q_{j}, w q_{k}, e_{1}\right\}$ can be extended to a matching, say, $M_{3}^{\prime}$, which covers the set $H$. Then the matching $M_{3}^{\prime}-u q_{j}-w q_{k}$ is a desired matching. Otherwise, the vertices $q_{j}$ and $q_{k}$ are adjacent. By Lemma 3.2, the 2-matching $\left\{q_{j} q_{k}\right\} \cup\left\{e_{1}\right\}$ can be extended to a matching, say, $M_{4}^{\prime}$, which covers the set $H$. Then the matching $M_{4}^{\prime}-q_{j} q_{k}$ is a desired matching.

When $x=0$, the vertex set $Q$ contains exactly three unfaithful vertices. Let $q_{j}$ be a vertex in $Q$ which is not unfaithful. Let $M_{5}$ be the perfect matching of the path $H-h_{j}$ of order $n-1$. Then, the matching $M_{5} \cup\left\{q_{j} h_{j}\right\}$ is a desired matching. This completes the proof of Lemma 3.3.

Now we deal with the first case $z=0$. Let $M$ be the matching obtained from Lemma 3.3. Let $M^{\prime}$ be the matching of the subgraph $G\left[J^{\prime}\right]$ which is symmetric to $M$. In other words, an edge $h_{i}^{\prime} h_{i+1}^{\prime}$ (resp. $\left.h_{j}^{\prime} q_{j}^{\prime}, q_{j}^{\prime} q_{j+1}^{\prime}\right)$ is in the matching $M^{\prime}$ if and only if the edge $h_{i} h_{i+1}$ (resp. $h_{j} q_{j}, q_{j} q_{j+1}$ ) is in $M$. Then the set

$$
M \cup M^{\prime} \cup\left\{q_{j} q_{j}^{\prime}: q_{j} \in J-V(M)\right\}
$$

is a perfect matching of the graph $G$ which covers the matching $M_{0}$, as desired.

Next lemma is for the case $(x, y, z)=(1,1,1)$.

Lemma 3.4. For any edge $e$ in the subgraph $G[J]$, and for any vertex $q_{k}$ in the set $Q-V(e)$, the subgraph $G[J]-V(e)-q_{k}$ has a perfect matching.

Proof. It suffices to find a matching $M$ of the subgraph $G[J]-V(e)-q_{k}$, such that

1. the path $G[H]-V(M)-V(e)$ is of even order;

2. every path component of the subgraph $G[Q]-V(M)-V(e)-q_{k}$ is of even order.

Below we will construct such a matching $M$ according to the position of the edge $e$.

Case 1. If $V(e) \subset H$, we can suppose that $e=h_{0} h_{1}$ without loss of generality, and take the matching

$$
M= \begin{cases}\left\{h_{2} q_{2}\right\}, & \text { if } k \text { is odd } \\ \left\{h_{2} q_{n+2}\right\}, & \text { if } k \text { is even }\end{cases}
$$


Case 2. If $V(e) \cap H \neq \emptyset$ and $V(e) \cap Q \neq \emptyset$, then we can suppose that $e=h_{1} q_{1}$ without loss of generality, and take the matching

$$
M= \begin{cases}\emptyset, & \text { if } k \text { is even } \\ \left\{h_{0} q_{0}, h_{2} q_{2}\right\}, & \text { if } k \text { is odd }\end{cases}
$$

Case 3. If $V(e) \subset Q$, then we can suppose $e=q_{0} q_{1}$ without loss of generality, and take the matching

$$
M= \begin{cases}\left\{h_{2} q_{2}\right\}, & \text { if } k \text { is odd } \\ \left\{h_{n-1} q_{2 n-1}\right\}, & \text { if } k \text { is even }\end{cases}
$$

This completes the proof.

Now we are ready to solve the case $x=y=z=1$. By Lemma 3.4, the subgraph $G[J]-V\left(M_{0}\right)$ has a perfect matching. For the same reason, the subgraph $G\left[J^{\prime}\right]-V\left(M_{0}\right)$ has a perfect matching. The union of these two matchings and the matching $M_{0}$ form a desired perfect matching of the graph $G$.

For the last case $(x, y, z)=(2,0,1)$, we will need the following lemma.

Lemma 3.5. Let $e_{0}$ be an edge of the subgraph $G[Q]$. Then any 2-matching of the subgraph $G[J]$ can be extended to a near perfect matching of $G[J]$, which covers the vertex set $H \cup V\left(e_{0}\right)$.

Proof. Let $\left\{e_{1}, e_{2}\right\}$ be a 2-matching of the subgraph $G[J]$. It suffices to show that the subgraph

$$
G[J]-V\left(e_{1} \cup e_{2}\right)
$$

has a matching $M$ such that

1. every path component of the subgraph $G[H]-V\left(e_{1} \cup e_{2} \cup M\right)$ is of even order;

2. at most one of the path components of the subgraph $G[Q]-V\left(e_{1} \cup e_{2} \cup M\right)$ is of odd order;

3. if the subgraph

$$
G[Q]-V\left(e_{1} \cup e_{2} \cup M\right)
$$

has an isolated vertex, then the isolated vertex is not an end of the edge $e_{0}$. 
If such a matching $M$ exists, then the subgraph $G[Q]-V\left(e_{1} \cup e_{2} \cup M\right)$ has a near perfect matching $M^{\prime}$, such that the matching $M \cup M^{\prime} \cup\left\{e_{1}, e_{2}\right\}$ covers the set $V\left(e_{0}\right)$. The desired result follows immediately. Below we will seek the above matching $M$. According to the positions of the edges $e_{1}$ and $e_{2}$, we have six cases to treat.

Case 1. Both the edges $e_{1}$ and $e_{2}$ are from the subgraph $G[H]$. The subgraph $G[H]-V\left(e_{1} \cup e_{2}\right)$ consists of two paths of different parities of orders, in which the path of even order might be empty. Let $h_{i}$ be an end of the path of odd order. We can take the matching $M=\left\{h_{i} q_{i}\right\}$.

Case 2. The edge $e_{1}$ is from the subgraph $G[H]$, and the edge $e_{2}=h_{j} q_{j}$ for some $j \in[2 n]$. We can suppose that $e_{1}=h_{0} h_{1}$ without loss of generality. Then we can take the matching

$$
M= \begin{cases}\left\{h_{j} q_{j}: 2 \leq j \leq n-1\right\}, & \text { if } e_{2} \in\left\{h_{j} q_{j}: 2 \leq j \leq n-1\right\}, \\ \left\{h_{j} q_{j}: n+2 \leq j \leq 2 n-1\right\}, & \text { otherwise, i.e., if } e_{2} \in\left\{h_{j} q_{j}: n+2 \leq j \leq 2 n-1\right\}\end{cases}
$$

Case 3. The edge $e_{1}$ is from the subgraph $G[H]$, and the edge $e_{2}$ is from the subgraph $G[Q]$. Without loss of generality, we can suppose that

$$
e_{2}=q_{0} q_{1} \quad \text { and } \quad e_{1}=h_{i} h_{i+1},
$$

where $0 \leq i \leq n-1$. Moreover, by symmetry, we can suppose that $0 \leq i \leq(n-1) / 2$ without loss of generality. We can take the matching

$$
M= \begin{cases}\left\{h_{i+2} q_{i+2}\right\}, & \text { if the vertex } q_{2} \text { is not an end of the edge } e_{0}, \\ \left\{h_{3} q_{n+3}\right\}, & \text { otherwise. }\end{cases}
$$

Case 4. Both the edges $e_{1}$ and $e_{2}$ have the form $h_{j} q_{j}$, where $j \in[2 n]$. Without loss of generality, we can suppose that $e_{1}=h_{1} q_{1}$. Then we can take the matching

$$
M= \begin{cases}\left\{h_{j} q_{j}: 2 \leq j \leq n\right\}, & \text { if } e_{2} \in\left\{h_{j} q_{j}: 2 \leq j \leq n\right\} \\ \left\{h_{j} q_{j}: n+2 \leq j \leq 2 n\right\}, & \text { otherwise, i.e., if } e_{2} \in\left\{h_{j} q_{j}: n+2 \leq j \leq 2 n\right\}\end{cases}
$$

Case 5. The edge $e_{1}$ has the form $h_{j} q_{j}$ for some $j \in[2 n]$, and the edge $e_{2}$ is from the subgraph $G[Q]$. Without loss of generality, we can suppose that $e_{2}=q_{0} q_{1}$ and $j \in[n]$, and take the matching

$$
M= \begin{cases}\left\{h_{2} q_{2}, h_{4} q_{4}\right\}, & \text { if } q_{2} \in V\left(e_{0}\right), \text { and } j=3 \\ \emptyset, & \text { otherwise. }\end{cases}
$$


Case 6. Both the edges $e_{1}$ and $e_{2}$ are from the subgraph $G[Q]$. The subgraph $G[Q]-V\left(e_{1} \cup e_{2}\right)$ consists of two paths, where one of them might be empty. Since the sum $2 n-4$ of their orders is even, the two paths have the same parity of orders. Since $2 n-4 \geq 6$, there is at most one path is of order 1 . If such an isolated vertex exists, say, $q_{j}$, then we can take the matching $M$ to be the edge $h_{j} q_{j}$. Otherwise, we can take the matching $M$ to be the edge $h_{k} q_{k}$, where $q_{k}$ is an end of the path of larger order, or an end of any path when the two paths have the same orders. This completes the proof of Lemma 3.5.

The last case $(x, y, z)=(2,0,1)$ can be done as follows. Let

$$
M_{0}=\left\{e_{1}, e_{2}, e_{3}\right\}
$$

where the edges $e_{1}$ and $e_{2}$ are faithful, and the edge $e_{3}$ is co-faithful. By Lemma 3.5, the subgraph $G[J]$ has a near perfect matching $M_{1}$ covering $\left\{e_{1}, e_{2}\right\}$, such that the associated uncovered vertex $q_{j}$ satisfies that its symmetric vertex $q_{j}^{\prime}$ is uncovered by $e_{3}$. By Lemma 3.4, the subgraph

$$
G\left[J^{\prime}\right]-V\left(e_{3}\right)-q_{j}
$$

has a perfect matching $M_{2}$. Hence the matching

$$
M_{1} \cup M_{2} \cup\left\{e_{3}, q_{j} q_{j}^{\prime}\right\}
$$

is a perfect matching of $G$ which extends $M_{0}$. This completes the proof of Theorem 3.3 .

Since the bow-tie graph $C_{6} \bowtie P_{n}$ can be embedded onto the Klein bottle, Theorem 3.3 implies immediately that there is an infinite number of 3-extendable graphs which are $\mathrm{N}_{2}$-embeddable. We further propose the following conjecture.

Conjecture 3.1. The graph $C_{m} \bowtie P_{n}$ is 3-extendable for any integers $m$ and $n$ such that $m$ is even.

\section{Proof of Theorem 1.2}

Recall that $\mu^{\prime}(\Sigma)$ is the minimum integer $k$ such that there is no $\Sigma$-embeddable $k$-extendable non-bipartite graphs. It follows that

$$
\mu^{\prime}(\Sigma) \leq \mu(\Sigma)
$$

This section is devoted to find out $\mu^{\prime}(\Sigma)$. We will need the following lemma. 
Lemma 4.1. Let $k \geq 1$. Any connected $k$-extendable graph of order $2 k+2$ is either the complete graph $K_{2 k+2}$, or the complete bipartite graph $K_{k+1, k+1}$.

Proof. It is easy to show for the case $k=1$. Below we let $k \geq 2$. Let $G$ be a connected $k$-extendable graph with $|G|=2 k+2$. By Theorem 2.8, either the graph $G$ is bipartite or we have $\kappa(G) \geq 2 k$.

In the former case, any vertex in the part with larger order has degree at most the order of the other part, and thus, at most $|G| / 2=k+1$. By Theorem 2.5, we have $\delta(G) \geq k+1$. Therefore, both parts of the graph $G$ has order $k+1$. Since $\delta(G) \geq k+1$, we infer that $G=K_{k+1, k+1}$.

In the latter case, we suppose to the contrary that the graph $G$ is not complete. Then $G$ has a pair $(u, v)$ of non-adjacent vertices. Since the graph $G$ is $k$-extendable and is of order $2 k+2$, we infer that the subgraph $G^{\prime}=G-u-v$ does not have a perfect matching. On the other hand, any graph $H$ of even order with $\delta(H) \geq|H| / 2$ has a Hamilton circuit; see Ore [13]. Since

$$
\delta\left(G^{\prime}\right) \geq 2 k-2 \geq 2 k / 2=\left|G^{\prime}\right| / 2
$$

we deduce that the subgraph $G^{\prime}$ has a Hamilton circuit, and a perfect matching in particular, a contradiction. This completes the proof.

Now we are in a position to show Theorem 1.2.

Proof. Let $\Sigma$ be a surface of characteristic $\chi$. Let $\mu^{\prime}(\Sigma)$ be the minimum integer $k$ such that every $\Sigma$-embeddable non-bipartite graph is not $k$-extendable.

First, we deal with the sporadic cases that $\chi \geq-1$. For the sphere $S_{0}$, by Ineq. (4.1),

$$
\mu^{\prime}\left(S_{0}\right) \leq \mu\left(S_{0}\right)=3
$$

by Theorem 1.1. On the other hand, it is clear that the graph $P_{4} \times C_{5}$ is planar and non-bipartite. Since it is 2-extendable by Theorem 3.1, we deduce that $\mu^{\prime}\left(S_{0}\right)=3$.

By Ineq. (4.1) and Theorem 1.1, $\mu^{\prime}\left(N_{1}\right) \leq \mu\left(N_{1}\right)=3$. Since every planar graph is $N_{1}$-embeddable, we deduce that $\mu^{\prime}\left(N_{1}\right) \geq \mu^{\prime}\left(S_{0}\right)=3$. Therefore, we conclude that $\mu^{\prime}\left(N_{1}\right)=3$.

For the torus $S_{1}$, by Ineq. (4.1) and Theorem 1.1,

$$
\mu^{\prime}\left(S_{1}\right) \leq \mu\left(S_{1}\right)=4 .
$$

On the other hand, it is clear that the graph $C_{6} \times C_{5}$ is toroidal and non-bipartite. Since it is 3 -extendable by Theorem 3.2 , we infer that $\mu^{\prime}\left(S_{1}\right)=4$. 
For the Klein bottle $N_{2}$, we have

$$
\mu^{\prime}\left(N_{2}\right) \leq \mu\left(N_{2}\right)=4
$$

by Theorem 1.1. On the other hand, the $N_{2}$-embeddable non-bipartite graph $C_{6} \bowtie P_{5}$ is 3-extendable by Theorem 3.3. Thus $\mu^{\prime}\left(N_{2}\right)=4$.

Along the same line, we have $\mu^{\prime}\left(N_{3}\right) \leq 4$. Since $\mu^{\prime}\left(N_{3}\right) \geq \mu^{\prime}\left(N_{2}\right)=4$, we infer that $\mu^{\prime}\left(N_{3}\right)=4$.

Below we can suppose that $\chi \leq-2$. Write

$$
n=\lfloor(7+\sqrt{49-24 \chi}) / 4\rfloor .
$$

Since $\chi \leq-2$, one may estimate that $n \geq 4$. By Theorem 2.3, it is direct to check that $K_{2 n}$ is $\Sigma$-embeddable. It is obvious that $K_{2 n}$ is both $(n-1)$-extendable and non-bipartite. Thus $\mu^{\prime}(\Sigma) \geq n$.

Suppose, by way of contradiction, that $\mu^{\prime}(\Sigma)>n$. Then there exists a the graph $G$, which is $\Sigma$-embeddable, $n$-extendable, and non-bipartite. The $n$-extendability implies that $|G| \geq 2 n+2$. If $|G|=2 n+2$, then $G=K_{2 n+2}$ by Lemma 4.1. By computing the genus and the non-orientable genus of $K_{2 n+2}$ directly, we see that $K_{2 n+2}$ is not $\Sigma$-embeddable. Thus we have $|G| \geq 2 n+4$.

Let $v$ be a control point in an embedding of the graph $G$ on $\Sigma$. Assume that $|G| \leq 4 n$. By Theorem 2.8, we deduce that the connectivity $\kappa(G)$ is at least $2 n$. It follows that

$$
d(v) \geq \delta(G) \geq \kappa(G) \geq 2 n
$$

Let $x$ be the number of triangles containing $v$. Let $y=d(v)$. When $y=2 n$, since $G$ is $n$-extendable, we infer that $x \leq 2 n-2$. By Lemma 2.1,

$$
\frac{2 n+1}{6}=\frac{y}{4}-\frac{2 n-2}{12} \leq \frac{y}{4}-\frac{x}{12} \leq 1-\frac{\chi}{2 n+4} .
$$

Solving it we find that $n \leq(1+\sqrt{81-24 \chi}) / 4$. By Eq. (4.2), we obtain that

$$
\frac{7+\sqrt{49-24 \chi}}{4}-1<\left\lfloor * \frac{7+\sqrt{49-24 \chi}}{4}\right\rfloor \leq \frac{1+\sqrt{81-24 \chi}}{4},
$$

which implies that $\chi>0$, a contradiction. Otherwise $y \geq 2 n+1$. By Lemma 2.1,

$$
\frac{2 n+1}{6} \leq \frac{y}{6} \leq 1-\frac{\chi}{2 n+4}
$$

which is same to Ineq. (4.3) and thus impossible. 
Now we are led to the case that $|G| \geq 4 n+2$. Assume that $x \leq 2 n-2$. By Lemmas 2.1 and 2.2 ,

$$
\frac{n+1}{4} \leq \frac{n+1+\lceil x / 2\rceil}{4}-\frac{x}{12} \leq \frac{y}{4}-\frac{x}{12} \leq 1-\frac{\chi}{4 n+2} .
$$

Solving it we find that $n \leq(5+\sqrt{49-16 \chi}) / 4$. By Eq. (4.2), we obtain that

$$
\frac{7+\sqrt{49-24 \chi}}{4}-1<\left\lfloor * \frac{7+\sqrt{49-24 \chi}}{4}\right\rfloor \leq \frac{5+\sqrt{49-16 \chi}}{4} .
$$

Solving the above inequality we find that $\chi \in\{-6,-5,-4,-3,-2\}$. Substituting each of these five values of $\chi$ into Ineq. (4.4), we obtain a contradiction. Otherwise $x \geq 2 n-1$. Then Lemma 2.1 gives

$$
\frac{n+1}{4} \leq \frac{2 n+1}{6}=\frac{y}{6} \leq 1-\frac{\chi}{4 n+2},
$$

the same contradiction. This completes the proof of Theorem 1.2.

At the end of this paper, we would like to share the approach of finding Eq. (1.2). Our previous result [12] on $(n, k)$-graphs is as follows.

Theorem 4.1 (Lu and Wang). Let $\Sigma$ be a surface of characteristic $\chi$. Let $\mu(n, \Sigma)$ be the minimum integer $k$ such that there is no $\Sigma$-embeddable $(n, k)$-graphs. Then for $n \geq 1$, we have

$$
\mu(n, \Sigma)= \begin{cases}\max (0,3-\lceil n / 2\rceil), & \text { if } \Sigma \text { is homeomorphic to the sphere, } \\ \max (0,\lfloor(7-2 n+\sqrt{49-24 \chi}) / 4\rfloor), & \text { otherwise. }\end{cases}
$$

While Eq. (4.5) was obtained by laborious computations, we discover Eq. (1.2) by the guess-and-check strategy. Although $(0, k)$-graphs are exactly $k$-extendable graphs, Eq. (4.5) is valid under the premise $n \geq 1$. Nevertheless, it was Eq. (4.5) by which we were inspired to guess Eq. (1.2) out.

\section{Acknowledgements}

The authors appreciate the anonymous referee for his/her careful reading and detailed corrections for improvement. Lu is supported by the National Natural Science Foundation of China (NSFC, Grant No. 11871391). Wang is supported by the NSFC (Grant No. 12171034) and the Fundamental Research Funds for the Central Universities (Grant No. 2021CX11012). He is grateful to the hospitality of Department of Mathematics in MIT. 


\section{References}

[1] R. E. L. Aldred, K. Kawarabayashi, and M. D. Plummer, On the matching extendability of graphs in surfaces, J. Combin. Theory Ser. B, 98 (2008), pp. 105-115.

[2] L. W. Beineke, R. J. Wilson, J. L. Gross, and T. W. Tucker, Topics in Topological Graph Theory, Cambridge University Press, 2009.

[3] N. Dean, The matching extendability of surfaces, J. Combin. Theory Ser. B, 54 (1992), pp. 133-141.

[4] P. Franklin, A six colour problem, J. Math. Phys., 13 (1934), pp. 363-369.

[5] J. L. Gross and T. W. Tucker, Topological Graph Theory, Wiley, 1987, and Dover, 2001.

[6] E. Györi and M. D. Plummer, The Cartesian product of a $k$-extendable and an $l$-extendable graph is $(k+l+1)$-extendable, Discrete Math., 101 (1992), pp. 87-96.

[7] J. Lakhal and L. Litzler, A polynomial algorithm for the extendability problem in bipartite graphs, Inform. Process. Lett., 65 (1998), pp. 11-16.

[8] H. Lebesgue, Quelques conséquences simples de la formule d'Euler, J. de Math., 9 (1940), pp. 27-43.

[9] J. Liu and Q. Yu, Matching extensions and products of graphs, Ann. Discrete Math., 55 (1993), pp. 191-200.

[10] D. Lou and Q. Yu, Connectivity of $k$-extendable graphs with large $k$, Discrete Appl. Math., 136 (2004), pp. 55-61.

[11] L. Lovász and M. D. Plummer, Matching Theory, Vol. 29, North-Holland Publishing Co., 1986.

[12] H. Lu and D. G. L. Wang, Surface embedding of $(n, k)$-extendable graphs, Discrete Appl. Math., 179 (2014), pp. 163-173.

[13] O. Ore, Note on Hamilton circuits, Amer. Math. Monthly, 67(1) (1960), 55.

[14] O. Ore, The Four-Color Problem, Academic Press, New York, 1967, pp. 54-61.

[15] O. Ore and M. D. Plummer, Cyclic colorations of plane graphs, in: W. T. Tutte (Ed.), Recent Progress in Combinatorics, Academic Press, New York, 1969, pp. 287-293.

[16] T. Parsons, G. Pica, T. Pisanski, and A. Ventre, Orientably simple graphs, Math. Slovaca, 37 (1987), pp. 391-394.

[17] M. D. Plummer, On n-extendable graphs, Discrete Math., 31 (1980), pp. 201-210.

[18] M. D. Plummer, Matching extension and the genus of a graph, J. Combin. Theory Ser. B, 44 (1988), pp. 329-337.

[19] M. D. Plummer, A theorem on matchings in the plane, in C. T. B. T. Lars Dovling Andersen, Ivan Tafteberg Jakobsen and P. D. Vestergaard (Eds.), Graph Theory in Memory of G. A. Dirac, vol. 41, Elsevier, 1988, 347-354.

[20] M. D. Plummer, Extending matchings in graphs: A survey, Discrete Math., 127 (1994), pp. 277-292.

[21] M. D. Plummer, Recent Progress in Matching Extension, in: Building Bridges, Bolyai Soc. Math. Stud., 19, M. Grötschel, G. O. H. Katona, G. Sági (Eds.), Springer Berlin Heidelberg, 2008, pp. 427-454. 
[22] G. Ringel, Bestimmung der Maximalzahl der Nachbargebiete auf nichtorientierbaren Flächen, Math. Ann., 127 (1954), pp. 181-214.

[23] G. Ringel and J. W. T. Youngs, Solution of the Heawood map-coloring problem, Proc. Natl. Acad. Sci. USA, 60 (1968), pp. 438-445.

[24] R. Rizzi, A short proof of König's matching theorem, J. Graph Theory, 33(3) (2000), pp. $138-139$.

[25] W.T. Tutte, The factorization of linear graphs, J. London Math. Soc., 22(2) (1947), pp. $107-111$.

[26] J. W. T. Youngs, Minimal imbeddings and the genus of a graph, J. Math. Mech., 12 (1963), pp. 303-315.

[27] Q. Yu and G. Liu, Graph Factors and Matching Extensions, Higher Education Press, Springer-Verlag, Berlin Heidelberg, 2009. 